\title{
Large-scale molecular-dynamics study of the nucleation process of martensite in Fe-Ni alloys
}

\author{
K. Kadau, P. Entel, T.C. Germann ${ }^{1}$, P.S. Lomdahl ${ }^{1}$ and B.L. Holian ${ }^{1}$ \\ Theoretische Tieftemperaturphysik, Gerhard-Mercator-Universität Duisburg, Lotharstrasse 1, \\ 47048 Duisburg, Germany \\ 1 Theoretical Division, Los Alamos National Laboratory, Los Alamos, NM 87545, U.S.A.
}

\begin{abstract}
High-performance large-scale molecular-dynamics (MD) simulations provide an atomistic insight of the nucleation process in $\mathrm{Fe}_{80} \mathrm{Ni}_{20}$. With the MD code SPaSM (Scalable Parallel Short-range Molecular Dynamics [1]) it is possible to follow the nucleation and further growth of the martensite structure (bcc) for more than one million atoms. The simulations show that the nucleation process is heterogeneous, at pre-existing defects. Further growth of the martensite structure into the austenite matrix (fcc) forms a typical twin structure on the nanoscale. Analysis of energy barriers between the martensite and the austenite can be used to interpret the nucleation process.
\end{abstract}

\section{INTRODUCTION}

Although research on martensitic materials has been done for about 100 years, many open questions still exist. The dynamics of nucleation processes is still not clear. There is a debate whether the martensitic transformation exists for small particles, and what drives this phenomenon. If the martensitic phase is suppressed in small particles, there might be two possible explanations: The phase stability is modified by surface effects, i.e., there might be a change in the free energy of the system due to different surface contributions of the two phases. The better established explanation is that the number of nucleation sites in small particles is too low compared to the bulk material. MD simulations $[2,3]$ show that it is necessary to introduce defects to obtain martensitic transformations in thin iron films and bulk iron nickel systems. Defects like vacancies allow a heterogeneous nucleation process [4], while rough films with islands compared to smooth films allow the system to transform because of the lack of lateral stresses [5]. This paper is focused on corners as nucleation sites in the $\mathrm{Fe}_{80} \mathrm{Ni}_{20}$ system as well as analysis of the nucleation process itself. To analyze the energetics of the fcc-bcc transformation, the Bain-transformation [6] is used, which creates the bcc structure by contracting one [001] axis of the fcc structure by $20.6 \%$ and stretching the two other [001] axes by $12.3 \%$. However, this transformation might not reproduce exactly the atomic path throughout the structural transformation. But in combination with a rotation it can reproduce the experimentally observed structural orientational relationships like Nishiyama-Wassermann, Kurdjumov-Sachs, or the Pitsch.

\section{COMPUTATIONAL METHODS}

The embedded-atom-method potential, which was developed and successfully used for several problems involving structural phase transition $[2,3,7]$, was used to describe interatomic forces in the Fe- $\mathrm{Ni}$ system. This many-body potential makes use of the form proposed by Daw and Baskes $[8,9]$ :

$$
E=\sum_{i} F_{\alpha_{i}}\left(\rho_{i}\right)+\sum_{i} \sum_{j \neq i} \frac{Z_{\alpha_{i}}\left(r_{i j}\right) Z_{\alpha_{j}}\left(r_{i j}\right)}{r_{i j}},
$$


where the indices $i$ and $j$ denote individual atoms; $\alpha_{i}, \alpha_{j}=\mathrm{Fe}, \mathrm{Ni}$ refer to the species of the corresponding atoms, and $r_{i j}$ is the separation of atoms $i$ and $j$. The function $F_{\alpha_{i}}$ represents the embedding energy of atom $i$ depending on the background charge density

$$
\rho_{i}=\sum_{j \neq i} \rho_{\alpha_{j}}^{a t}\left(r_{i j}\right)
$$

which is a superposition of the contributions $\rho_{\alpha_{j}}^{\text {at }}$ of all atoms $j$ other than $i$. The second term in Eq. (1) is a screened Coulomb potential with the effective charges $Z_{\alpha_{i}}$. The functions $F_{\alpha_{i}}$ and $Z_{\alpha_{i}}$ are cubic spline functions which have been fitted to specific properties of Fe and $\mathrm{Ni}$.

To study this system, we used MD simulations [10] to solve Newton's equations of motion for atoms or molecules. This tool gives insight into dynamical atomic processes, including chemical reactions. The simulations are carried out for a fixed number of particles $\mathrm{N}$ with or without periodic boundary conditions. In order to control the temperture, we made use of the Nosé-Hoover thermostat $[11,12]$. The calculation of absolute free energies is a very difficult numerical task [13], even in a stable state, and it is too difficult to calculate the entropy along the Bain path. Therefore, we analyzed the thermo-dynamical stability by calculating free energy differences for the fcc and bcc phase along the Bain path, by using the total differential of the free energy $F(T, V)[14]$ :

$$
\mathrm{d} F=-P \mathrm{~d} V-S \mathrm{~d} T
$$

$P$ is the pressure, $V$ is the volume, $S$ is the entropy, and $T$ is the temperature of the system. By simulating at constant temperature and writing the pressure in Cartesian components Eq. (3) leads to:

$$
\mathrm{d} F=-P_{x x} A_{y z} \mathrm{~d} x-P_{y y} A_{z x} \mathrm{~d} y-P_{z z} A_{x y} \mathrm{~d} z,
$$

where $P_{i i}$, denotes the pressure components along the three Cartesian axes and $A_{i j}$, denotes the respective perpendicular cross section.

\section{SIMULATION RESULTS}

The thermo-dynamical stability of the martensite and austenite phase is determined by simulating a rather small system (about 1000 atoms) of $\mathrm{Fe}_{80} \mathrm{Ni}_{20}$ at constant temperature with periodic boundary conditions. By calculating the pressure components $[4,15]$ and the energy along the Bain path, we obtained with equation (4) the free energy along the transformation path. The left panel of Figure 1 shows the energy as a function of the $c / a$ ratio with a minimum for the martensite phase at low temperatures and a minimum for the fcc phase at high temperatures. This could lead to the assumption that there is no large hysteresis, but in fact there is [16], and defects are needed to obtain a martensitic phase transformation. The trend of the free energy (right panel of Figure 1) is quite different from the energy curves: The austenite state is stable down to low temperatures, and there is an energy barrier between the fcc and the bcc state. This explains why there is no transformation to the bcc phase if there are no defects: The system is not able to overcome the energy barrier between the two states by collective motion. It rather has to nucleate at defects where the energy barrier is locally reduced.

To analyze the dynamics of such a nucleation process we simulated a cubic nano-particle with an edge length of $24 \mathrm{~nm}$ containing slightly more than one million atoms. Starting with a perfect fcc structure with the [001] axes parallel to the edges of the nano-particle and free boundary conditions, we performed simulations for 75 ps (50,000 integration steps), using the SPaSM [1] code on an 8processor $250 \mathrm{MHz}$ SUN E4000 parallel computer system at Los Alamos. The CPU time for the 75 ps was about $250 \mathrm{~h}$.

Figure 2 illustrates the time evolution of martensitic nucleation and further growth into the austenitic matrix. Half of the system is shown, in order to give insight into the interior of the particle during this 
Energy

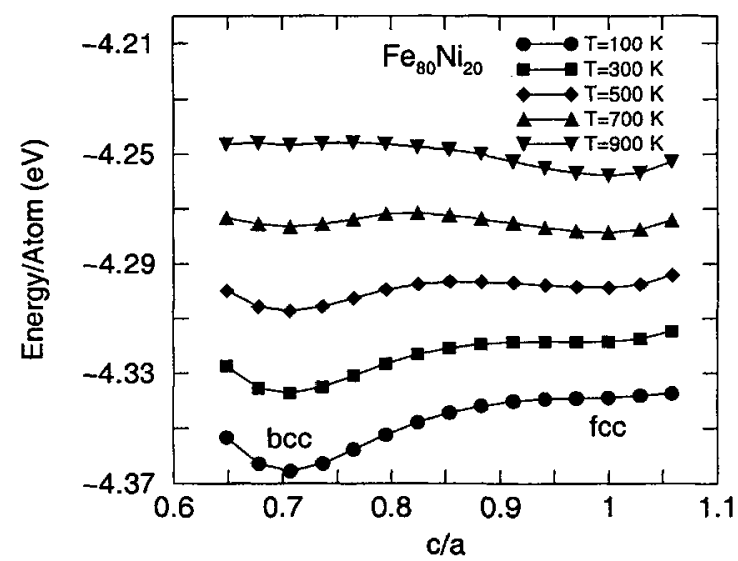

Free Energy

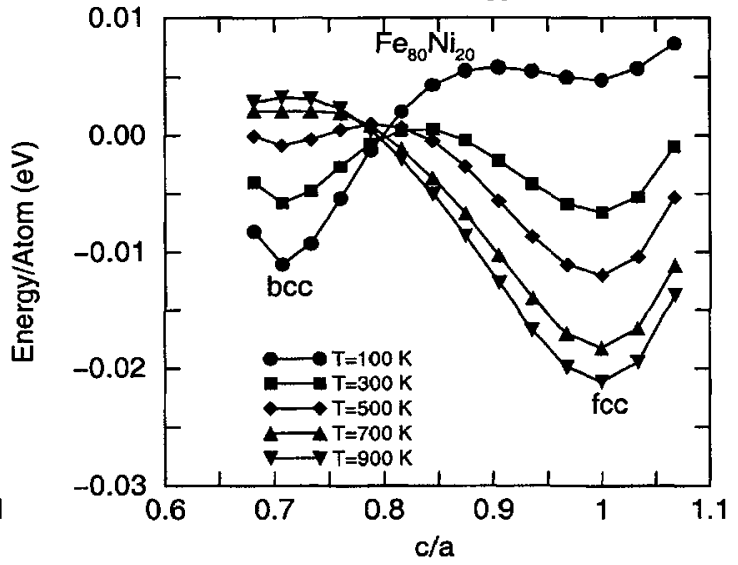

Figure 1: Energy and free energy along the Bain-path for $\mathrm{Fe}_{80} \mathrm{Ni}_{20}$ for different temperatures. Left panel: The minimum of the energy at low temperaures for the bcc structure gradually vanishes with increasing temperature; the fcc minimum appears at high temperatures. However, note that the energy is not the correct quantity to determine thermodynamic stability of a canonical system. Right panel: The minimum of the free energy for the bcc state also vanishes at high temperatures, while the fcc structure becomes more stable. In contrast to the energy, there remains a minimum of the free energy for the fcc structure at low temperatures which creates a metastable state of the fcc phase at low temperatures. The system has to overcome an energy barrier to complete the transition from fcc to bcc.

process. The martensite starts to nucleate at the corners of the cubic particle, where the coordination number is reduced as are, therefore, the energy barriers. Statistical thermal fluctuations can lead to nucleation at some corners rather than others. The surface begins to tilt, indicating a shear-like transformation; further growth into the core of the particle forms regions of martensite and austenite arranged in a needle like pattern. There is competition between the different martensitic variants nucleating from different corners, leading to different sizes of the variants. A closer inspection (Figure 4) of the final structure of the nano-particle reveals the separation of different grain orientations by twin boundaries. The initially fcc(001) surfaces transform either to a bcc(001) or a bcc(011) surface with a kink as the boundary for the different surface variants. In the interior of the resulting system the twin boundaries are much better pronounced, because of the lack of surface defects. The gradual transformation of austenite to martensite in time can be well demonstrated by the time evolution of the radial distribution function [10]. The first neighbor peak of the fcc structure (12 nearest neighbors) splits up into the two nearest neighbor peaks of the bcc structure ( 8 nearest and 6 second nearest neighbors) (Figure 3).

Similar MD simulations show that spherical nano-particles do not transform if there are no initially introduced defects like vacancies or partially missing planes. This strengthens the argument that pre-existing defects are needed for martensitic transformations in iron alloys.

\section{SUMMARY AND CONCLUSION}

Our simulations show that nucleation sites for iron-based alloys might play a major role in martesitic transformations. The analysis of the free energy along the transformation path reveals an energy barrier between the fcc and the bcc structure up to lowest temperatures. This explains the heterogeneous nucleation at defects and the growth of martensitic domains starting from defects like corners. 

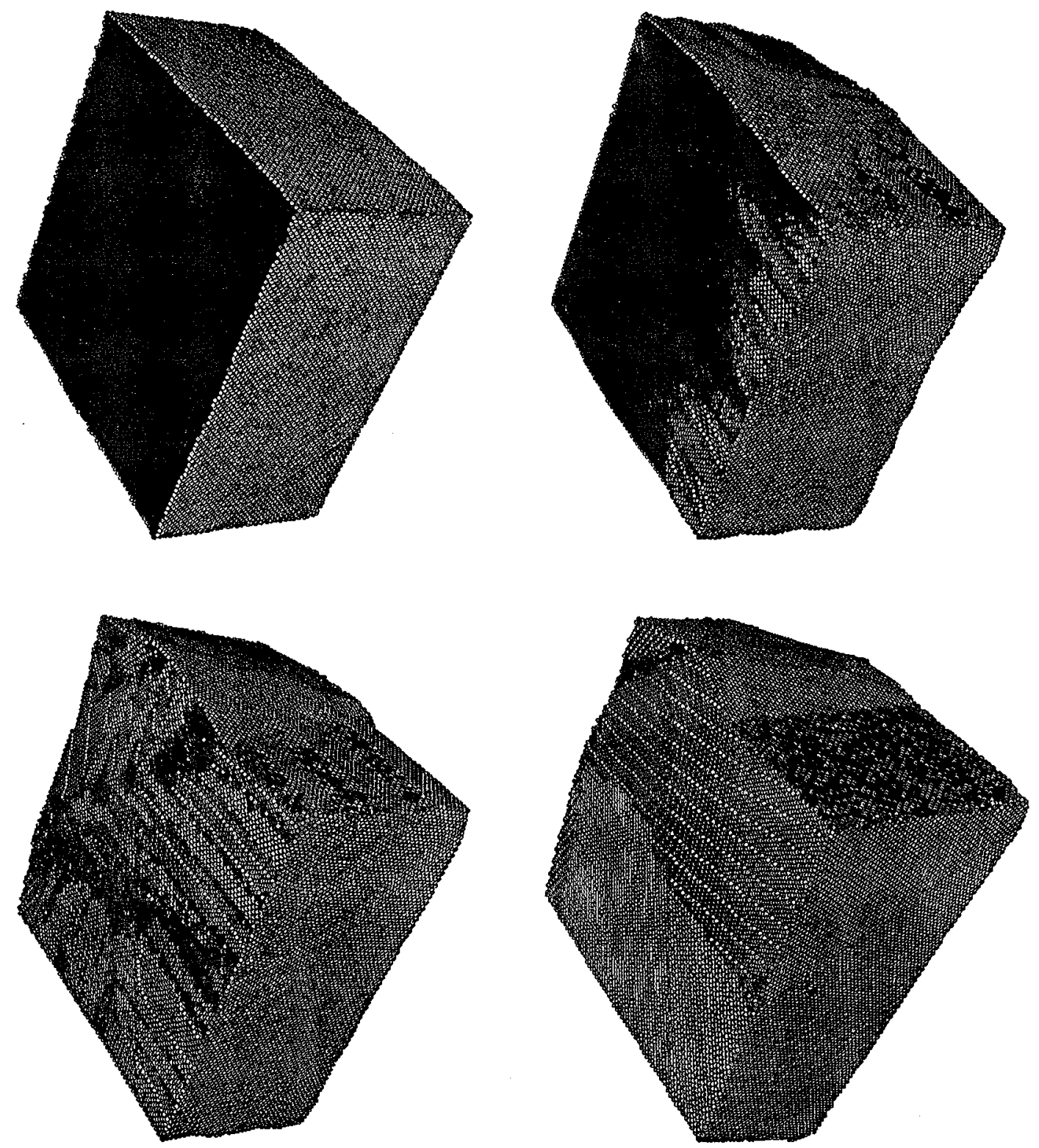

Figure 2: Time evolution of half of the initial fcc ordered simulation cell ( $3 \mathrm{ps}, 21 \mathrm{ps}, 33 \mathrm{ps}, 60 \mathrm{ps}$; left-right and top-bottom) at $\mathrm{T}=50 \mathrm{~K}$. The grey scale refers to the local coordination number of individual atoms (dark=12 neighbors or 4 neighbors, i.e., austenite or $b c c(001)$ surface), light $=8$ neighbors, i.e., martensite or fcc(001) surface). The martensite starts to nucleate at corners and surfaces of the crystal. Statistical thermal fluctuations cause asymmetry in nucleation sites. After 33 ps martensite (light) and austenite (dark) form a needle-like pattern, and the orginally flat surface has some kinks, which result in different shear directions and therefore crystallographic orientations of the martensite. Finally, the crystal is nearly completely transformed into different martensitic domains, separated by twin boundaries. 

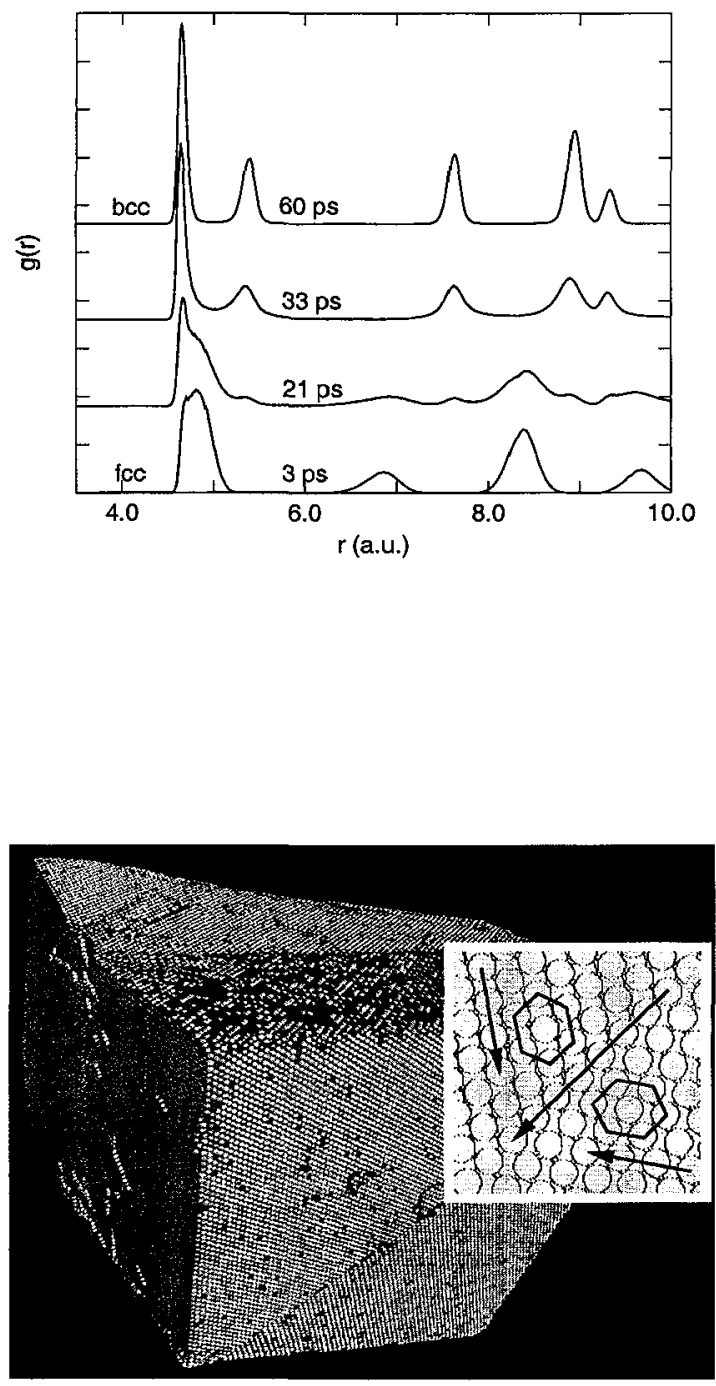

Figure 3: Time evolution of the radial distribution function of a cubic particle of $\mathrm{Fe}_{80} \mathrm{Ni}_{20}$ containing one million atoms. The gradual transformation from $\mathrm{fcc}$ to bcc at $\mathrm{T}=50 \mathrm{~K}$ can be seen by the splitting of the first neighbor peak of the fcc structure (12 nearest neighbors) into the two nearest neighbor peaks of the bcc structure ( 8 nearest and 6 second nearest neihgbors).

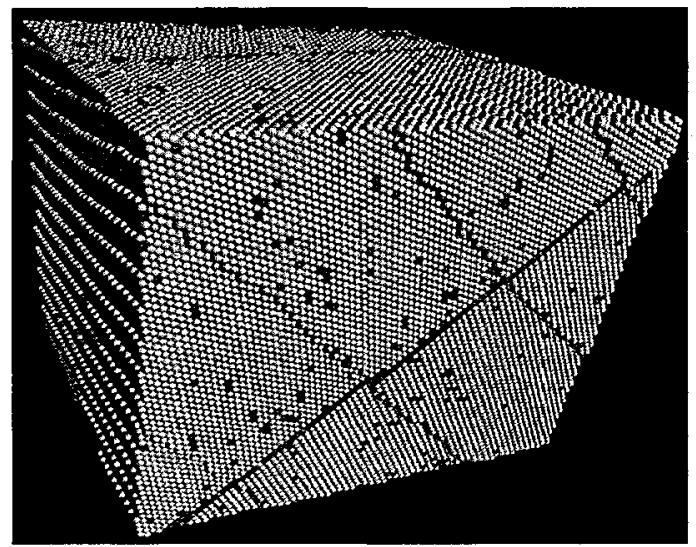

Figure 4: Final structure of the nano-particle. The grey scale refers to the local coordination number of individual atoms varying from 4-12 for the left panel and from 8-12 the right panel. Left panel: The tilt between the (001) surface (dark) and the (011) surface (light) is due to a shear transformation typical for martensite. The front surface shows a twinning system of the bcc structure (see inset): The long arrow indicates a [111]-direction, the short arrows marks the [001] directions of the two twinned variants of the bcc phase. The distorted hexagons are typical for the bcc(011) surface. Right Panel: The interior of the finally obtained martensite block shows the twin boundaries better because of the lack of surface defects: One along the diagonal of the front surface, and the other one extending from the tilt in the surface. The other less-straight lines are due to cutting the system along planes with high indices. 
The final martensite structure is twinned, and the initially flat surface is tilted, which reflects the shear-like martensitic transformation on a nanoscale.

\section{Acknowledgements}

One of the authors (K.K.) would like to thank Ralf Meyer (Duisburg) and Christian Helm (Los Alamos) for many helpful and fruitful discussions. This work was carried out under the auspices of the U.S. Department of Energy and the German Science Council (DFG) through the Graduate School "Structure and Dynamics of Heterogeneous Systems" and SFB 445.

\section{References}

1. David M. Beazley and Peter S. Lomdahl, Computers in Physics 11, 230 (1997).

2. K. Kadau, R. Meyer and P. Entel, Surf. Rev. Lett. 6, 35 (1999).

3. P. Entel, K. Kadau, R. Meyer, H. C. Herper, M. Schröter and E. Hoffmann, Phase Transitions 65, 79 (1998).

4. P. Entel, R. Meyer and K. Kadau, Philos. Mag. B 80, 183 (2000).

5. K. Kadau, R. Meyer and P. Entel, Structure and Dynamics of Heterogeneous Systems edited by P. Entel and D. E. Wolf (World Scientific, Singapore 2000).

6. E. C. Bain and N. Y. Dunkirk, Trans. AIME, 70, 25 (1924).

7. R. Meyer and P. Entel, Phys. Rev. B 57, 5140 (1998).

8. M. S. Daw and M. I. Baskes, Phys. Rev. Lett. 50, 1285 (1983).

9. M. S. Daw and M. I. Baskes, Phys. Rev. B 29, 6443 (1984).

10. M. P. Allen and D. J. Tildesley, Computer Simulation of Liquids (Clarendon Press, Oxford, 1987).

11. W. G. Hoover, Phys. Rev. A 31, 1695 (1985).

12. S. Nosé, Mol. Phys. 52, 255 (1984).

13. P. Entel, R. Meyer, K. Kadau, H. C. Herper and E. Hoffmann Eur. Phys. J. B 5, 379 (1998).

14. L. D. Landau and E. M. Lifshitz, Statistical Physics, 3rd edn. (Pergamon Press, Oxford, 1993).

15. U. Landman, Wear 153,1 (1992).

16. P. Entel, K. Kadau, R. Meyer, H.C. Herper, M. Acet, E.F. Wassermann, J. Magn. Magn. Mater., 177-181, 1409 (1998). 